\title{
Physical dependency of elderly and physically disabled; measurement concordance between 10 -item Barthel index and 5-item shorter version
}

\author{
S Lekamwasam ${ }^{1}$, K Karunatilake ${ }^{2}$, S K P Kankanamge², V Lekamwasam ${ }^{2}$
}

(Index words: validation of questionnaire, Barthel Index, Sri Lanka)

\begin{abstract}
Introduction Barthel index consisting 10 items is used to estimate physical dependence of elderly and physically disabled. A shorter version with 5 items has been developed and it is more suitable as a rapid screening tool of physical dependence.
\end{abstract}

Objectives To assess the measurement agreement between 10-item Barthel index and 5-item shorter version.

Methods The 10-item Barthel index was translated to Sinhala, adhering to the standard protocols and validated among 286 patients with varying degree of physical disability selected by stratified quota system. From the same data sheets, scores given for bathing, transfer, toileting, walking and climbing steps were used to make the shorter version.

Results The 5-item Barthel index showed a high internal consistency (global Cronbach's alpha $=0.93$ ). The 10 -item version also showed a similar internal consistency (global Cronbach's alpha $=0.92$ ) while its item-total correlations varied between $0.64-0.90$ for all items except for the urinary and bladder functions. In the factor analysis, urinary and bowel functions factored together and independent to other items and these two factors accounted for $73 \%$ variation of the score. The total scores of the 10-item and 5-item versions showed a high correlation $(r=0.9, p<0.001)$. In the Bland-Altman plot, more than $95 \%$ of data points were within the $\pm 1.96 \mathrm{SD}$ tolerance limits.

Conclusions This analysis illustrates the reliability and validity of the Sinhala version of 10 -item Barthel index in estimating physical activities of daily living and the high measurement concordance between the standard 10 -item and 5-item shorter versions.

Ceylon Medical Journal 2011; 56: 114-118

${ }^{1}$ Department of Medicine, Faculty of Medicine, Galle, Sri Lanka, ${ }^{2} T e a c h i n g$ Hospital, Karapitiya, Sri Lanka.

Correspondence: SL, e-mail: <slekamwasam@gmail.com>. Received 7 January 2011 and revised version accepted 27 April 2011. Competing interests: none declared. 


\section{Introduction}

The proportion of elderly in the population has shown a steady increase over the past years and this trend is especially noticeable in Sri Lanka [1]. Elderly are prone to debilitating illnesses which are associated with functional impairment which needs to be quantified for their initial assessment and monitoring during follow up. Functional assessment is often ignored in busy clinical set ups especially when elderly are treated in units which are not dedicated for elderly care.

Self-administered questionnaires are used to identify those with high risk for certain conditions such as angina, colorectal carcinoma and upper gastrointestinal diseases. Questionnaires used to measure physical dependence and quality of life is, generally, self-administered. Unlike interviewer-administered questionnaire, a self-administered questionnaire eliminates observer bias and inter-observer variability. In addition they are relatively inexpensive and require no professional time. Barthel index (BI) measures physical activities of daily living (PADL), an estimate frequently used in the rehabilitation of patients with chronic debilitating illnesses. Mahoney and Barthel in 1965 introduced the 10-item BI as a measure of physical disability or dependence and is available at http://www.strokecenter.org/trials/scales/ barthel.pdf [2]. This self-administered questionnaire consists of 10 items, each addressing different domains of PADL. They include feeding, bathing, grooming, dressing, bowel and bladder functions, toilet use, transfer (moving between bed and chair), walking on flat surface and using steps.

Although the original version of BI consists of 10 items [2], attempts have been made to increase the number of items to 15 [3] and 17 [4] to enhance the sensitivity of the tool. In the 10 -item version, the total score varies between $0-100$ with 0 indicating the worst physical activity and 100 representing complete physical independent state. Patients could be further categorised to totally dependent (score between 0-20), severely dependence (score between 21-60), moderately dependent (score between 61-90) and slightly dependent (score between 91-99) based on the baseline value $[3,5]$.

Both the original and modified versions of BI have shown a high degree of reliability [3, 6]. Attempts have also been made earlier to reduce the number of items to 5 to make the BI more user-friendly and the questionnaire has been translated to other languages such as Chinese and Turkey $[7,8,9]$. The shorter version of BI could be incorporated in the clinical assessment of elderly during the history taking and used as a screening tool to assess functional dependence. This is particularly important in Sri Lanka as elders are treated in general medical wards which have a rapid patient turnover and are not dedicated to elderly needs. Adaptation of a questionnaire to accommodate ethnic and language variations is necessary to increase its applicability in a wider population. This analysis was designed to validate the Sinhala version of $\mathrm{BI}$ and to examine the measurement concordance between the standard 10-item version and 5-item shorter version.

\section{Methods}

This research proposal was approved by the Ethics Review Committee of the Faculty of Medicine, Galle. First, two bilingual medical postgraduates, fluent in both English and Sinhala, translated the 10-item version of the BI to Sinhala language, independently. They made sure that no additions, omissions or alterations were done to the original content of items. They, together with two authors (SL and KK), combined the two translations to one single questionnaire to maximise the clarity of items. Two other postgraduates with the same language skills back translated the Sinhala version to assess the comparability with the original version. Once the clarity, understandability and naturalness of items were assessed in a focused group discussion comprised of eight staff nurses and four medical students, the questionnaire was piloted among 30 participants with chronic illnesses selected from medical wards. The Sinhala version of BT (10-item BI-S) was finalised after the preliminary analysis of the pilot study.

The 10-item BI-S was given to 286 particpants, selected by stratified quota method based on age and comorbidity. Inclusion criteria were age between $40-80$, conversant in Sinhala language and ability to read and write without assistance. In order to capture a heterogeneous group with regards to their physical activities, the participants were selected from medical and orthopaedic clinics. As instructed in the original version, participants were advised to record what they did and not what they could do, based on their daily activities over the past one week.

Reliability of the 10-item BI-S was tested using intraclass correlation (item-total) and Cronbach's alpha for internal consistency. Construct validity was examined by factor analysis using the principal component analysis as the extraction method and Varimax with Keiser normalisation as the rotation method. The number of factors was determined by setting the Eigen value greater than 1.

From the same data sheets, scores given for five items; bathing, toileting, transfer, walking and climbing steps, were selected and the 5-item shorter version (5-item BI-S) was constructed. This shorter version has been used in previous studies [7]. Internal consistency of 5-item BI version was tested using intra-class correlation (item-total) and Cronbach's alpha. 
Correlation between the total scores of the 10-item and 5-item versions was examined using Pearson correlation. A Bland-Altman plot was constructed with difference between the two total scores in the y axis and average of the two total scores in the $\mathrm{x}$ axis. Limits of tolerance were defined as mean \pm 1.96 standard deviations around the average value. Furthermore, a categorical analysis was done by cross tabulating the quartiles of the total scores of the two versions to test for inter-rater agreement and kappa value.

\section{Results}

Out of 286 participants recruited, 120 of them were attendees of medical clinics for various long standing medical conditions (diabetes, heart failure or ischaemic heart disease). Fifty nine had suffered from strokes while others had fractures of long bones, 6 months or before. Age of the participants ranged from $45-78$ with a median of 68 years and 102 of them were males.

Study participants found the items in the 10-item BI$\mathrm{S}$ questionnaire clear and easily understandable. Most were able to answer the questionnaire within five minutes and without assistance. Of 286, 58 (20.3\%) were fully independent and scored the maximum points (ceiling) while $6(2 \%)$ were fully physically incapacitated and scored minimum possible (floor). Items addressing bathing and grooming scored the minimum while transfer and mobility showed higher scores (Table 1).
Table1. Characteristics of the study sample $(n=286)$

\begin{tabular}{lc}
\hline Character & \multicolumn{1}{c}{ Value } \\
\hline Mean (inter-quartile range) age: years & $68(62-71)$ \\
Number (proportion) of males & $102(47.2 \%)$ \\
Number (proportion) of subjects with & \\
Maximum score $(100)$ & $58(20.3 \%)$ \\
Minimum score $(0)$ & $6(2 \%)$ \\
\hline
\end{tabular}

\section{Reliability}

In reliability analysis, 10 -item $\mathrm{BI}-\mathrm{S}$ version showed a high internal consistency (global Cronbach's alpha $=0.92$, $p<0.001$ ). Removal of a particular item did not materially improve the internal consistency. Bowel and urinary continence showed poor item-total correlations $(0.39$ and 0.28 , respectively) while other items showed higher (range 0.64-0.90) item-total correlations (Table 2). The 5 -item BI-S version too showed a high internal consistency (global Cronbach's alpha $=0.93, p<0.001$ ) and removal of a particular item did not improve the alpha value (data not shown).

Table 2. Reliability and validity testing of Sinhala version of 10-item Barthel Index

\begin{tabular}{lccccc}
\hline Item & Mean (SD) & Factor 1 & Factor 2 & ITC & alpha \\
\hline Feeding & $7.1(3.9)$ & 0.73 & & 0.64 & \\
Bathing & $2.8(2.5)$ & 0.92 & & 0.83 & \\
Grooming & $3.0(2.5)$ & 0.88 & & 0.81 & \\
Dressing & $6.4(4.4)$ & 0.88 & & 0.82 & \\
Bowels & $9.2(2.2)$ & - & 0.78 & 0.39 & 0.92 \\
Bladder & $9.0(2.5)$ & - & 0.85 & 0.28 & \\
Toilet use & $6.3(4.3)$ & 0.89 & & 0.86 & \\
Transfers & $10.1(6.3)$ & 0.87 & & 0.90 & \\
Mobility & $10.1(6.6)$ & 0.78 & & 0.80 & \\
Stairs & $5.8(4.6)$ & 0.80 & & 0.78 & \\
\hline
\end{tabular}

Data extraction using principal component analysis with Varimax rotation. Factor loading $<0.30$ is suppressed for presentation. ITC $=$ item-total correlation, alpha $=$ Cronbach's alpha 


\section{Validity}

Only the 10-item BI-S version was tested for validity. In factor analysis, KMO value was 0.87 with Bartlett's significance of $<0.001$. Two factors emerged with Eigen values greater than 01 and accounted for $73 \%$ of variation of the score. The factor loading for the two factor solution is shown in Table 2. Urinary and bladder continence factored together and different to other eight items. The factor number of two was supported by the Scree plot which showed levelling of the plot at two factor level.

\section{Measurement concordance between the two versions}

The total scores of the 10-item BI-S and 5-item BI-S versions showed a high correlation $(\mathrm{r}=0.97, p<0.001)$. In the Bland-Altman plot only 12 observations were beyond the 1.96 SD tolerance limits indicating a good measurement agreement between the two versions. Most of the outliers were in the lower end of the plot indicating that the disagreement is mainly confined to the lower scorers (Figure). When the quartiles of the total scores of the two versions were cross tabulated (Table 3 ), an inter-rater agreement of 0.86 was found. The kappa values for the agreement was $0.81(\mathrm{SE}=027, p<0.001)$.

Table 3. Agreement between quartiles of the 10-item and 5-item versions of the Barthel index

\begin{tabular}{lllll}
\hline Variables & 10-item BI & 10-item BI & 10-item BI & 10-item BI \\
Quartile 1 & Quartile 2 & Quartile 3 & Quartile 4
\end{tabular}

$\begin{array}{lcccc}\text { 5-item BI Quartile 1 } & 66 & 6 & 0 & 0 \\ \text { 5-item BI Quartile 2 } & 6 & 57 & 9 & 0 \\ \text { 5-item BI Quartile3 } & 0 & 5 & 44 & 14 \\ \text { 5-item BI Quartile 4 } & 0 & 0 & 0 & 79\end{array}$

Inter-rater agreement $=0.86, \mathrm{kappa}=0.81(\mathrm{SE}=0.027, p<0.001)$

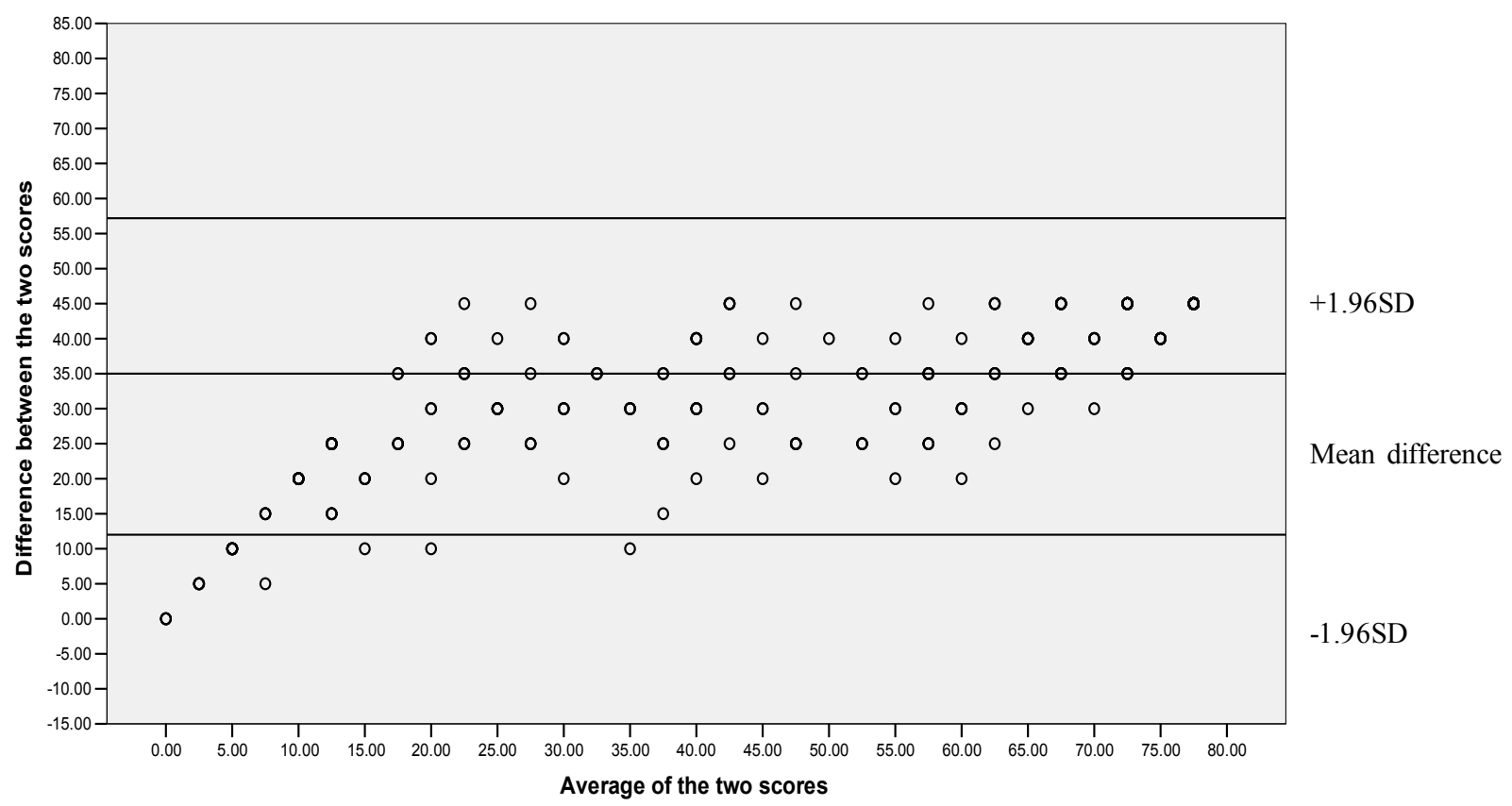

Figure. Bland-Altman plot for the measurement agreement between the total scores of the two versions. 


\section{Discussion}

This study measured agreement between the standard 10-item BI and modified 5-item shorter version when assessing physical dependence of elderly and physically disabled. During the process we were able to examine the psychometric properties of the Sinhala translation. The Sinhala translation of the 10-item BI we used, showed a high reliability, ie. high internal consistency (global Cronbach's alpha $=0.92$ ). Our results are consistent with those observed for the 15-item version (alpha of 0.95) and for the 10-item version (alpha 0-87-0.92) [3, 6, 7, 8].

Reliability indicates the extent of the random error associated with a measuring instrument and Cronbach's alpha which assesses the internal consistency is a better indicator of the reliability in a multi-item questionnaire [10]. A minimum alpha value of 0.8 has been recommended when group comparisons are made while a minimum of 0.95 has been recommended when clinical decisions are made at individual level [11]. We observed an alpha value of 0.92 which is very close to the 0.95 cut-off value recommended for individual patient assessment. In this analysis, all items except bowel and bladder functions showed high item-total correlations and similar results have been reported earlier. Hobart and Thompson also found a relatively low item-total correlations for bowel and bladder functions ( 0.34 and 0.42 , respectively) while correlations of other items varied from 0.53 to 0.83 [7].

In the factor analysis, we observed two factors. Urinary and bowel habits factored together and separately from other eight items. Similar observation has been made when the Chinese version of the BI was validated [8]. While items related to urinary and bowel habits measure physiological functions related to sphincters, the remaining factors reflect motor functions. This possibly explains why these two items behave differently from other factors, and have low item-total correlations. Nevertheless, bowel and bladder functions test a major domain in the functional assessment of elders and have clear implications in the long-term rehabilitation of patients with physical disabilities.

Our study sample consisted of patients with varying physical abilities; patients with chronic illnesses who were physically independent (patients attending medical clinics) and patients following either a major stroke or fracture. In the sample, six patients had the lowest possible score while 58 patients had the highest possible score. This would increase the reliability of the analysis as it allowed the questionnaire to be tested among a group of patients with a wide range of physical disability. Furthermore, this analysis showed the ability of 5-item BI-S to be used as rapid screening test to detect those who need a comprehensive assessment of PADL. This will help busy clinicians to detect those with impaired physical functions during history taking. Five items included in the shorter version can be incorporated in to routine history taking and if an elderly is found to have functional limitations in those items, he can be assessed further with 10-item standard BI.

This study has several limitations. Validation process involved a cross-sectional data collection and we have not followed up these patients to assess the long-term reliability of the questionnaire. Also the validation included patients between ages 40-80 and applicability of results to patients outside these age limits is unknown. Furthermore, the number of patients with total disability was relatively less.

Despite these limitations, this analysis indicates the high reliability of the Sinhala version of the BI when measuring the PADL in the local population and the ability of using the BI shorter version as a rapid screening test to detect those with impaired physical functions during their clinical assessment.

\section{References}

1. De Silva WI. Population projections for Sri Lanka 19912041. Institute of Policy Studies (Colombo). Vol 2, 1997.

2. Mahoney FI, Barthel DW. Functional evaluation: the Barthel index. Maryland State Medical Journal 1965; 14: 61-5.

3. Granger CV, Dewis LS, Peters NC, Sherwood CC, Barrett JE. Stroke rehabilitation: analysis of repeated Barthel index measures. Archives of Physical Medicine and Rehabilitation 1979; 60: 14-7.

4. McGinnis GE, Seward ML, DeJong G, Osberg JS. Programme evaluation of physical medicine and rehabilitation departments using self-report Barthel. Archives of Physical Medicine and Rehabilitation 1986; 67: 123-5.

5. Granger CV, Sherwood CC, Greer DS. Functional status measures in a comprehensive stroke care programme. Archives of Physical Medicine and Rehabilitation 1977; 58: 555-61.

6. Shah S, Vanclay F, Cooper B. Improving the sensitivity of the Barthel index for stroke rehabilitation. Journal of Clinical Epidemiology 1989; 42: 703-9.

7. Hobart JC, Thompson AJ. The five item Barthel index. Journal of Neurology Neurosurgery and Psychiatry 2001; 71: 225-30.

8. Leung SO, Chan CC, Shah S. Development of a Chinese version of the modified barthel index: validity and reliability. Clinical Rehabilitation 2007; 21: 912-22.

9. Kucukdeveci AA, Yavuzer G, Tennant A, Suldur N, Sonel B, Arasil T. Adaptation of the modified Barthel index for use in physical medicine and rehabilitation in Turkey. Scandinavian Journal of Rehabilitation Medicine 2000; 32: 87-92.

10. Stewart AL, Ware JE, eds. Measuring Functioning and Wellbeing: the Medical Outcomes Study Approach. Durham, North Carolina: Duke University Press, 1992.

11. Nunnally JB, Bernstein IH. Psychometric Theory, 3rd ed, McGraw Hill Inc, New York 1994. 